\title{
A l'Heure des Statecharts et de XPC target pour la Commande d'une Montre Digitale
}

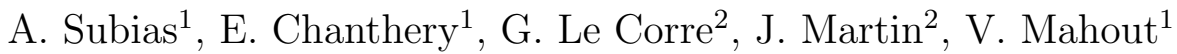 \\ subias@laas.fr \\ ${ }^{1}$ CNRS ; LAAS ; 7, avenue du Colonel Roche, F-31077 Toulouse, France \\ Université de Toulouse; UPS, INSA, INP, ISAE; LAAS ; F-31077 Toulouse, France \\ ${ }^{2}$ INSA; 135 avenue de Rangueil 31077 Toulouse France
}

RESUME : Cet article présente un projet développé au Département Génie Electrique et Informatique de l'Institut National des Sciences Appliquées de Toulouse (INSA) dont le but est la commande d'une montre digitale. La modélisation de la commande s'appuie sur les Statecharts et sa mise en œuvre repose sur l'utilisation d'un PC sous noyau temps-réel. Ce projet permet ainsi d'illustrer à la fois des enseignements de base en systèmes à événements discrets et des aspects plus avancés d'informatique industrielle.

\section{Introduction}

La création d'une manipulation de travaux pratiques est un problème multi objectifs très contraint. Aux objectifs pédagogiques évidents, peut s'ajouter celui de disposer d'un démonstrateur qui puisse intéresser le public étudiant visé, mais également le public moins averti des journées portes-ouvertes et autres visites de département, qui au travers de ce démonstrateur se fera sa propre idée d'un domaine scientifique voire même d'un futur métier.

C'est dans cette optique qu'a été développée au département Génie Electrique et Informatique de l'INSA de Toulouse la Montre Digitale. Le public étudiant concerné par ce projet est constitué des étudiants de niveau $L 2$ de la préorientation Mathématiques Informatique et Communication destinés aux filières Informatique et Réseaux, Génie Mathématique et Modélisation, et des étudiants de niveau L3 de la Préorientation Ingénierie des Matériaux, Composants et Systèmes s'orientant vers les filières Automatique et Electronique, Génie Physique et Ingénierie Systèmes. Ces deux publics étudiants recoivent dans un premier temps une formation théorique de base en logique combinatoire et séquentielle. A cette formation de base s'ajoute une formation théorique d'une douzaine heures orientée Systèmes à Evénements Discrets (SED) qui est illustrée par un cycle de travaux pratiques dans lequel s'insère le projet présenté ici. Le cours et les travaux dirigés abordent classiquement, les machines à états $(M A E)$, les réseaux de Petri, le GRAFCET et les Statecharts. Le cycle de travaux pratiques a pour objectif d'illustrer les problèmes de commande de SED et est constitué de trois séances de $2 \mathrm{H} 45$. Compte tenu du faible volume horaire imparti à cette formation, il est apparu pertinent de mettre en place trois manipulations dressant un panorama des outils de modélisation et des techniques de mise en oeuvre de la commande. Une première manipulation pose un problème de commande de tri de pièces sur la base d'une modélisation par réseau de Petri et d'une implémentation sur un API de type TSX Premium via une liaison ethernet. La seconde manipulation est un problème de commande de cabine d'ascenseur avec une modélisation par $M A E$ et une réalisation sur une carte FPGA de type Spartan 3 sous le logiciel Xilinx ISE. Pour la dernière manipulation il s'agit donc d'une commande d'une montre digitale avec une modélisation par Statecharts et une mise en oeuvre sur noyau temps réel. Ces trois manipulations permettent également d'établir des liens avec des enseignements connexes de VHDL, de réseaux de Petri, langage C, microcontrôleurs, etc...

Le choix de la montre digitale a été fait pour différentes raisons dont la première est son adéquation à une modélisation par Statecharts comme l'a présenté Harel [HAREL, 1988]. La montre est également une illustration capable d'intéresser des étudiants se destinant à des filières variées, abordable par un public non averti et présentant un caractère ludique ce qui est un atout supplémentaire. Le faible coût de réalisation de la maquette, le faible encombrement et la robustesse d'une telle maquette ont permis de créer quatre exemplaires du projet et d'accueillir chaque année environ 150 étudiants. 
Le projet présenté ici a été amorcé dans le cadre d'un projet tutoré de niveau $M 1$ (environ 60h). De cette manière il a été possible de définir des fonctions montre appropriées et d'évaluer plusieurs solutions tant au niveau de la conception de la maquette que de l'implémentation de la commande.

La suite de cet article est organisée de la manière suivante : la section 2 est dédiée à la montre. Le projet pédagogique est décrit dans la section 3 . Les objectifs visés par ce projet, la démarche suivie par les étudiants et le retour d'expérience y sont présentés. Enfin, la section 4 conclut en présentant les prochaines évolutions du projet.

\section{La montre}

L'étude et la réalisation de la maquette de la montre se sont faites entièrement au sein de l'atelier de mécanique et d'électronique de l'INSA pour un coût unitaire de 300 euros environ. Après conception d'un prototype, quatre exemplaires de la montre ont été réalisés et sont maintenant en service dans les salles de travaux pratiques $S E D$ du département Génie Electrique et Informatique de l'INSA de Toulouse.

\subsection{Cahier des charges}

La montre développée a les mêmes fonctionnalités qu'une montre digitale classique comme celle donnée sur la Figure 1.

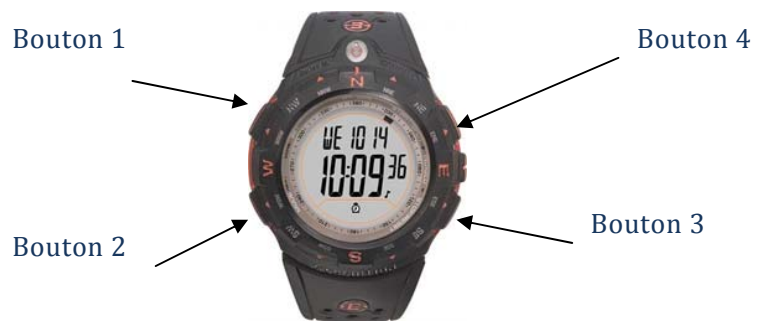

Figure 1 - Montre de base

Cette montre comporte plusieurs modes de fonctionnement (heure, date, alarme, chronomètre) accessibles par les quatre boutons Bouton $_{1}$, Bouton 2 , Bouton $_{3}$ et Bouton 4 ). Le fonctionnement de chaque bouton est le suivant :

- Bouton 4 : permet d'éclairer l'écran. Il peut être actionné en même temps que n'importe quel autre bouton.

- Bouton 3 : un appui simple sur ce bouton permet de passer de mode en mode de manière cyclique. Le mode initial est le modeheure, le mode suivant est le mode date, puis alarme, et enfin chronomètre.

- Bouton 2 : ce bouton permet, pour les modes heure, date et alarme de rentrer dans le mode réglage. Une fois le mode réglage sélectionné, un appui sur le Bouton $_{2}$ permet de passer à l'élément suivant à régler. Si le mode heure est actif, un appui sur le Bouton 2 permet de rentrer dans le mode réglage. Il faut alors régler d'abord les secondes. Ensuite, un nouvel appui sur le Bouton 2 permettra de régler les minutes, puis les heures, etc. de manière cyclique. Si le mode date est actif, le Bouton 2 permettra de régler le jour, le mois puis le jour de la semaine.

- Bouton $_{1}$ : lorsque le mode réglage est actif, ce bouton permet en général d'incrémenter de 1 l'élément à régler (sauf pour les secondes qui sont réinitialisées sur appui du Bouton 1 ).

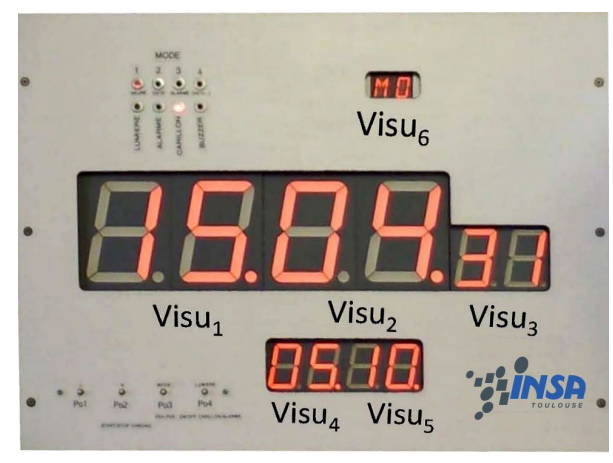

Figure 2 - Face avant de la montre

L'affichage des informations (voir Figure 2) se fait de la manière suivante : les digits principaux (au centre visu 1 à 3) affichent l'élément correspondant au mode de fonctionnement actif. Les digits du haut (visu 6) affichent toujours les deux premières lettres du jour (en anglais). Les digits des secondes en mode heure n'affichent rien dans les autres modes, sauf en mode chronomètre où ils permettent d'afficher les dixièmes et les centièmes de secondes. Par exemple, si le mode alarme est actif, l'heure et les minutes de l'alarme seront affichées sur les digits centraux. Initialement, le jour de la semaine est MOnday, la date 01.01 et l'heure 00.00.00.

Pour la gestion de l'alarme plusieurs choix sont disponibles. Il existe en effet 3 modes d'alarme.

- Carillon : un bip est émis par la montre à chaque passage d'une heure;

- Alarme : la montre sonne et le voyant buzzer s'allume à l'heure réglée par l'utilisateur ;

- Le dernier mode combine Carillon et Alarme. Pour régler ces modes, il faut appuyer sur Bouton $_{2}$ puis sur Bouton 4 tout en maintenant 
le premier bouton.

L'accès à ces trois modes se fait de manière cyclique : Bip horaire, Bip horaire et Alarme, puis Alarme, puis aucun des deux modes. Un appui sur un bouton quelconque permet d'arrêter la sonnerie de l'alarme. Deux voyants indiquent le mode d'alarme actif.
La montre dispose également d'une fonction chronomètre simplifiée. Une fois le mode chronomètre activé, l'appui sur le Bouton 2 permet de démarrer et de stopper le chronomètre. La remise à zéro s'effectue avec Bouton 1 .

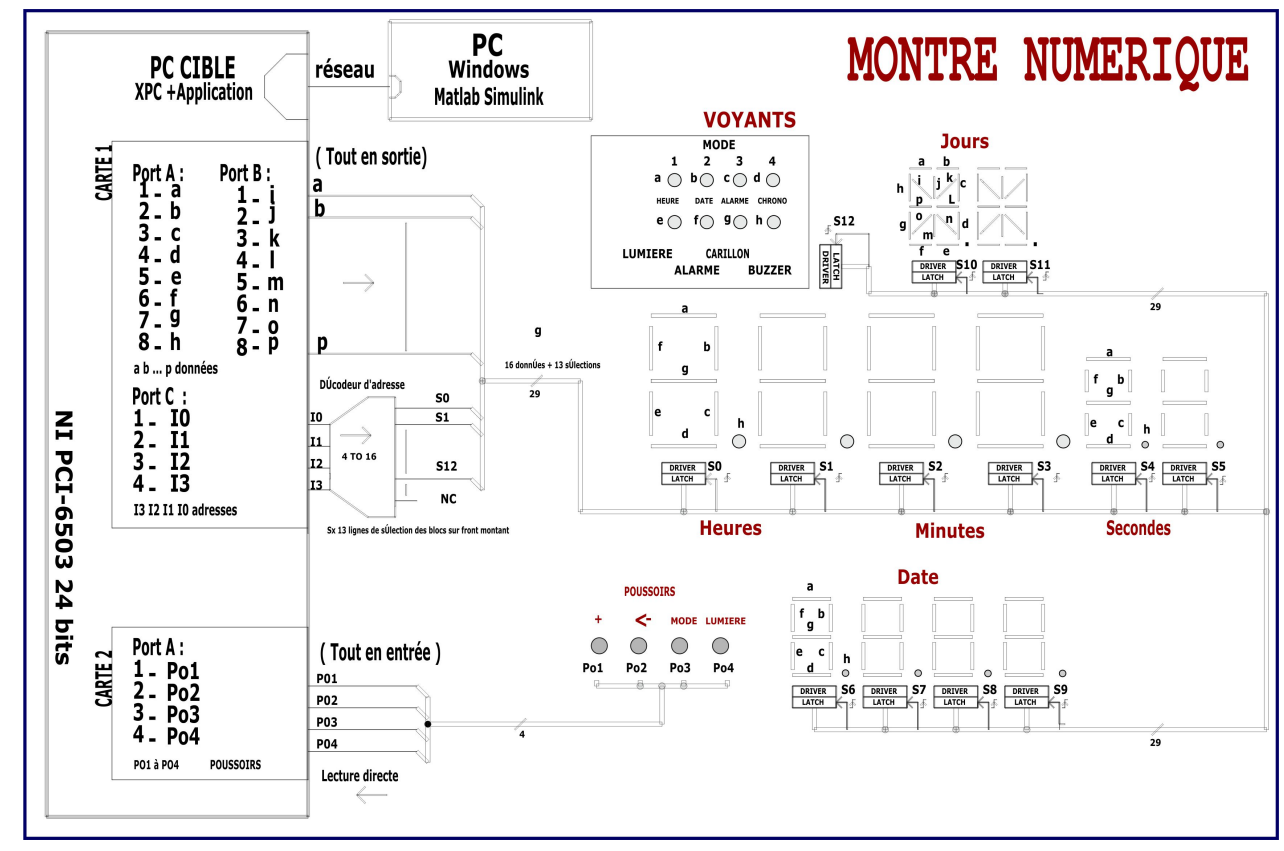

Figure 3 - Schéma fonctionnel de la montre

\subsection{La maquette de la montre}

La maquette développée s'appuie sur un support en bois rectangulaire taillé dans un grand format bien visible et fonctionnel. Cette maquette se compose d'afficheurs, 7 segments ou alphanumériques de différentes tailles, de boutons poussoirs et de voyants. Une vue arrière de la maquette (Figure 4) montre les cartes électroniques, les liaisons filaires, les bus et les alimentations.

L'électronique de la montre reste volontairement classique et se compose de circuits logiques de la famille 74 (bascule $\mathrm{D}$, décodeur), de buffers de courants, de transistors ...

Comme le montre le schéma fonctionnel (Figure 3), l'électronique a été étudiée pour permettre à l'élève la plus grande simplicité de mise en œuvre. L'affichage des chiffres et des lettres n'est pas multiplexé pour ne pas avoir à gérer le rafraîchissement en fréquence (rémanence de l'œil et luminosité). Chacun des blocs d'afficheurs ou de voyants garde en mémoire la dernière valeur reçue : l'inconvénient est que l'on multiplie le nombre de drivers de courant et de cir- cuits mémoires à bascules D. Chaque bloc est adressable par 4 lignes (I3 I2 I1 I0) qui permettent après décodage la sélection (S0 à S12) d'un afficheur ou de l'ensemble des voyants. Le bus de données se compose de 16 lignes $(a, b, \ldots p)$ de sorties qui pilotent chacune des segments des afficheurs ou des voyants. La lecture des boutons poussoirs est directe sur un deuxième bus de données (P01 P02 P03 P04).

L'interfaçage électrique de la montre avec le PC cible se fait par des cartes entrées/sorties (compatible Matlab/XPC Target) comportant un port numérique 24 bits. Chaque port ne pouvant être configuré, dans l'environnement XPC Target, que dans une direction donnée (entrée ou sortie) l'utilisation de deux cartes a été nécessaire.

Comme mentionné précédemment, la face avant de la maquette de la montre (Figure 2) comporte cinq zones de visualisation composées chacune de deux afficheurs sept segments et une sixième zone d'affichage composée de deux afficheurs alpha-numériques. La maquette comprend également quatre boutons correspondant à Bouton 1 , Bouton B $_{2}$, 
Bouton $_{3}$ et Bouton 4 . Huit voyants lumineux permettent de visualiser l'activation de la lumière de la montre, de l'alarme, du carillon, du buzzer ainsi que l'activation du mode de fonctionnement. Les grandeurs $V i s u_{x}$ correspondent aux nombres qui vont être affichés sur les afficheurs de la maquette et qui varient selon le mode de fonctionnement actif.

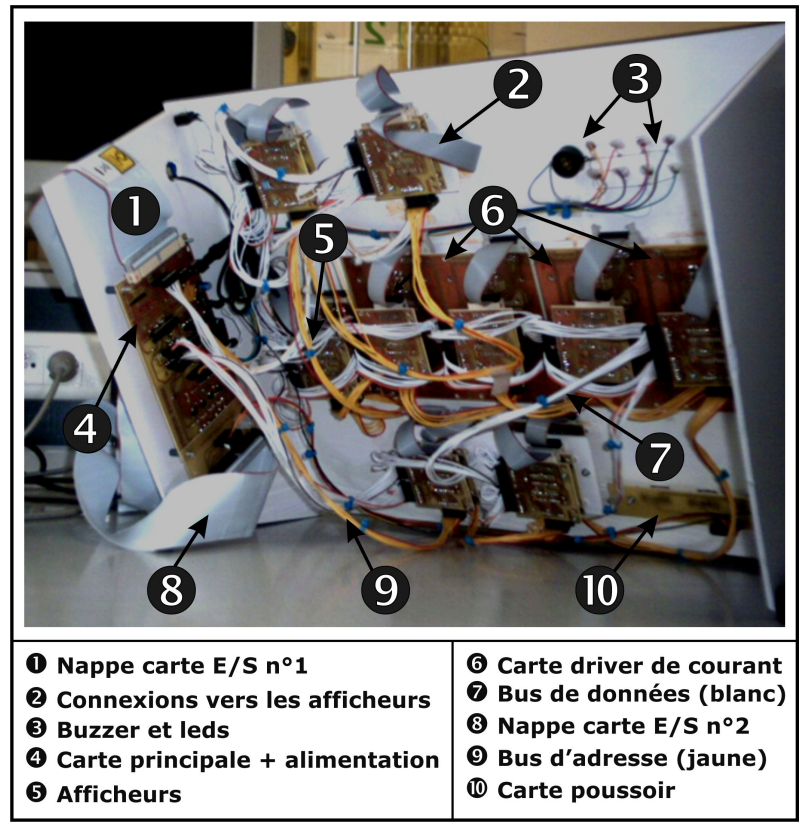

FiguRE 4 - Face arrière de la montre

\subsection{Commande sous XPC Target}

La commande de la montre est réalisée à l'aide d'un PC sous l'environnement XPC Target. XPC Target est une boîte à outils spécifique de Matlab qui permet de mettre au point, de tester et de mettre en œuvre des applications temps réel sur du matériel de type PC directement à partir de schémas Simulink.

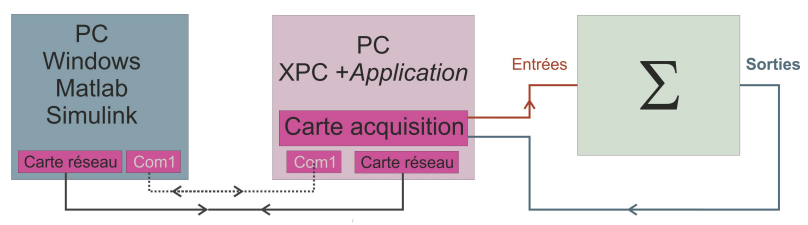

Figure 5 - Illustration de la commande déportée

Cet outil offre ainsi l'avantage de passer de la phase essai/simulation à la phase mise en œuvre/expérimentation très rapidement et très facilement en s'affranchissant de l'étape de codage. La configuration matérielle standard est composée de 2 unités informatiques reliées par une liaison de type ethernet (une communication par liaison série, plus lente, est également envisageable). Cette configuration nécessite également la possession de la boîte à outils Real Time Workshop $(R T W)$ de Matlab qui permet la traduction des schémas Simulink en code $\mathrm{C}$ ainsi qu'un compilateur C compatible avec l'ensemble du processus de développement (Microsoft Visual Studio dans notre cas)

- Le PC hôte sert d'unité de développement. A partir de Matlab/Simulink, il est possible d'engendrer directement l'application temps réel (commande de la montre dans notre cas). Il fonctionne dans l'environnement Windows habituel.

- Le PC cible est orienté temps réel. Il est donc démarré (à l'aide d'une EPROM) non pas sur DOS ou Windows mais sur le noyau temps réel spécifique XPC. Ce noyau, entre autres choses, permet de recevoir et de faire exécuter le code engendré par le PC hôte et d'assurer la communication entre les deux machines (téléchargement, mise en marche, adaptation en ligne de paramètres, rapatriement de données...). Enfin ce PC est équipé de cartes (dans notre cas deux cartes National Instrument PCI-6503 24 bits avec convertisseurs analogique/numérique et convertisseurs numérique/analogique) qui lui permettent de commander le processus.

La cible est équipée d'un moniteur vidéo sur lequel sont visualisés deux types d'information : des informations liées à l'exécution temps réel de l'application en cours (nom de l'application, temps courant, période d'échantillonnage...) ainsi qu'un rapport sur l'exécution des commandes envoyées par le PC hôte (adaptation de paramètres, mise en marche,...). Le second groupe d'informations est graphique et dépend de l'application. La Figure 5 présente les trois entités manipulées en TP : le PC de développement avec Matlab/Simulink et la boîte à outils Stateflow, le $\mathrm{PC}$ cible et le système à contrôler i.e. la maquette de la montre.

Le choix de cette architecture de commande est avant tout dicté par le souci d'efficacité dans le processus de codage ce qui permet aux étudiants de se concentrer sur l'objectif principal de cette étude, à savoir la manipulation des Statecharts. Cette solution offre également l'avantage d'établir un lien avec des microprojets en place au niveau $M 1$ de la filière Automatique et Electronique et qui s'appuient sur XPC Target pour de la commande de process. D'autres solutions ont tout de même été étudiées. La première était à base d'un produit Dspace. Cette alternative relativement équivalente à celle retenue sur le plan développement ne l'est pas d'un point de vue financier... La seconde consistait à utiliser des microcontrôleurs (Infinéon C167) sur lesquels les étudiants ont déjà travaillé dans le cadre d'appren- 
tissage en informatique matérielle. Ne disposant pas à l'époque de la licence du générateur de code Stateflow coder [MATLAB AND Simulink, ] cette solution nécessitait de "sortir" de Matlab pour inclure le code $\mathrm{C}$ engendré par RTW dans le programme du microcontrôleur et son logiciel de développement spécifique. Bien que ne présentant pas de difficulté majeure, cette seconde possibilité n'a pas eu de suite car elle nécessitait trop de manipulation de code.

\section{Le projet pédagogique}

\subsection{Objectifs pédagogiques visés}

Le premier objectif visé par ce projet est l'emploi des Statecharts comme outils de modélisation pour la commande d'un $S E D$. Le Statechart principal de la Montre Digitale est constitué de 8 macro-états ET aux travers desquels les principaux concepts de base des Statecharts que sont la hiérarchie, le parallélisme et la diffusion, sont illustrés :

- Gest_horloge gère le fonctionnement de l'horloge i.e.l'incrémentation des centièmes, secondes, minutes et heures.

- Gest_alarme gère l'activation du buzzer de l'alarme.

- Gest_date gère le fonctionnement de la date i.e. l'incrémentation du jour, mois et année.

- Mode_alarme gère le passage entre les trois modes d'alarme.

- Gest_bip_passe_heure gère l'activation du carillon de l'alarme.

- MODES1 permet d'effectuer les différents réglages de la montre et contient 2 sous états OU : TIME qui permet le réglage de l'heure et DATE qui permet le réglage de la date.

- Clignotement gère le clignotement des afficheurs en cours de réglage

- Gestion_Affichage assure l'affectation des données à afficher sur les différents afficheurs de la maquette en fonction du mode dans lequel se trouve la montre.

Ces différents macro-états permettent ensuite d'illustrer différents types de transitions :

- Transition avec condition du type [minutes $==60]$ dans l'état Gest_horloge ou [mois !=12] pour Gest_date

- Transition avec condition et action par exemple [Bouton $1=1 \quad \& \&$ Bouton $2=0 \quad \& \&$ minutes $<59 /$ minutes ++ ] pour le réglage des minutes dans l'état TIME.

- Transition avec événement seul : par exemple after(50,change(centiemes)) qui permet de détecter 50 occurrences successives de l'événement lié au changement de valeur de la variable centiemes. Ce type d'événement peut être utilisé pour gérer la durée du bip horaire par exemple.

Des actions au niveau des états peuvent aussi être facilement mises en place. Par exemple dans l'état Gest_alarme, une action du type entry :Bip $=1$ permet d'activer la sonnerie de l'alarme à l'activation de l'état dans lequel cette action est placée.

La modélisation est réalisée sous Matlab/Simulink avec la boîte à outils Stateflow [MAtlab And Simulink, ]. Les étudiants ont une connaissance de Matlab et de Simulink variable selon leur profil et leur niveau, mais aucun ne connaît Stateflow. Le projet permet ainsi de leur faire découvrir par apprentissage cette nouvelle boîte à outils qu'ils peuvent être amenés à réutiliser au cours de leur formation selon leurs choix d'orientation.

Le deuxième objectif pédagogique visé par ce projet est d'initier les étudiants à une commande sur une cible temps réel dans la mesure où ces aspects sont au programme des années suivantes de niveau $M 1$ et $M 2$. Cette initiation permet d'illustrer les notions de commande déportée, de PC hôte/cible, de cartes d'acquisition et autres concepts de base, mais permet aussi d'aborder des aspects de communication asynchrones importants.

\subsection{Démarche étudiante}

Le sujet de travaux pratiques développé autour de la Montre Digitale est calibré pour être réalisé par les étudiants en $2 H 45$. Pour cela, les étudiants disposent dès le départ d'une version simplifiée de la Montre Digitale. Il s'agit dans un premier temps de leur faire tester certaines fonctionnalités de base de la montre déjà modélisées. Cette première étape leur permet d'appréhender toutes les phases de la mise en ouvre de la commande au travers des actions de configuration de l'environnement logiciel XPC Target dans un premier temps, puis des actions liées à la compilation du source Simulink et des actions liées à l'execution du programme sur le $P C$ cible.

Dans un deuxième temps il leur est demandé de rajouter d'autres fonctionnalités. La première fonction à rajouter est une fonction Eclairage. Il est demandé aux étudiants de modifier le source initial en insérant un nouvel état et de faire les modifications nécessaires au niveau des entrées/sorties sous Simulink. Cette fonction Eclairage choisie pour sa simplicité (un macro état OU, 2 sous états ET, une transition sur condition d'appui d'un bouton) permet aux étudiants très rapidement de faire un cycle complet de développement. Cette fonction sert aussi 
de prétexte pour illustrer des actions de type During exécutées tant que l'état concerné est actif (activation de la lampe tant que Bouton 4 appuyé). La seconde fonction que les étudiants doivent mettre en place est la fonction Reglage_alarme. Cette fonction a été choisie en raison de sa similarité avec les fonctions de réglage de l'heure et de réglage de la date déjà en place dans le source Simulink fourni aux étudiants.
Les étudiants s'inspirent donc des modélisations fournies pour réaliser leur fonction Reglage_alarme. Par la suite, il est demandé aux étudiants selon la même démarche de mettre en place une fonction Chronometre en s'inspirant là encore d'un bloc Statechart déjà existant : Gest_horloge qui assure la gestion de l'horloge. La figure 6 illustre le bloc Statechart du chronomètre que peuvent développer les étudiants.

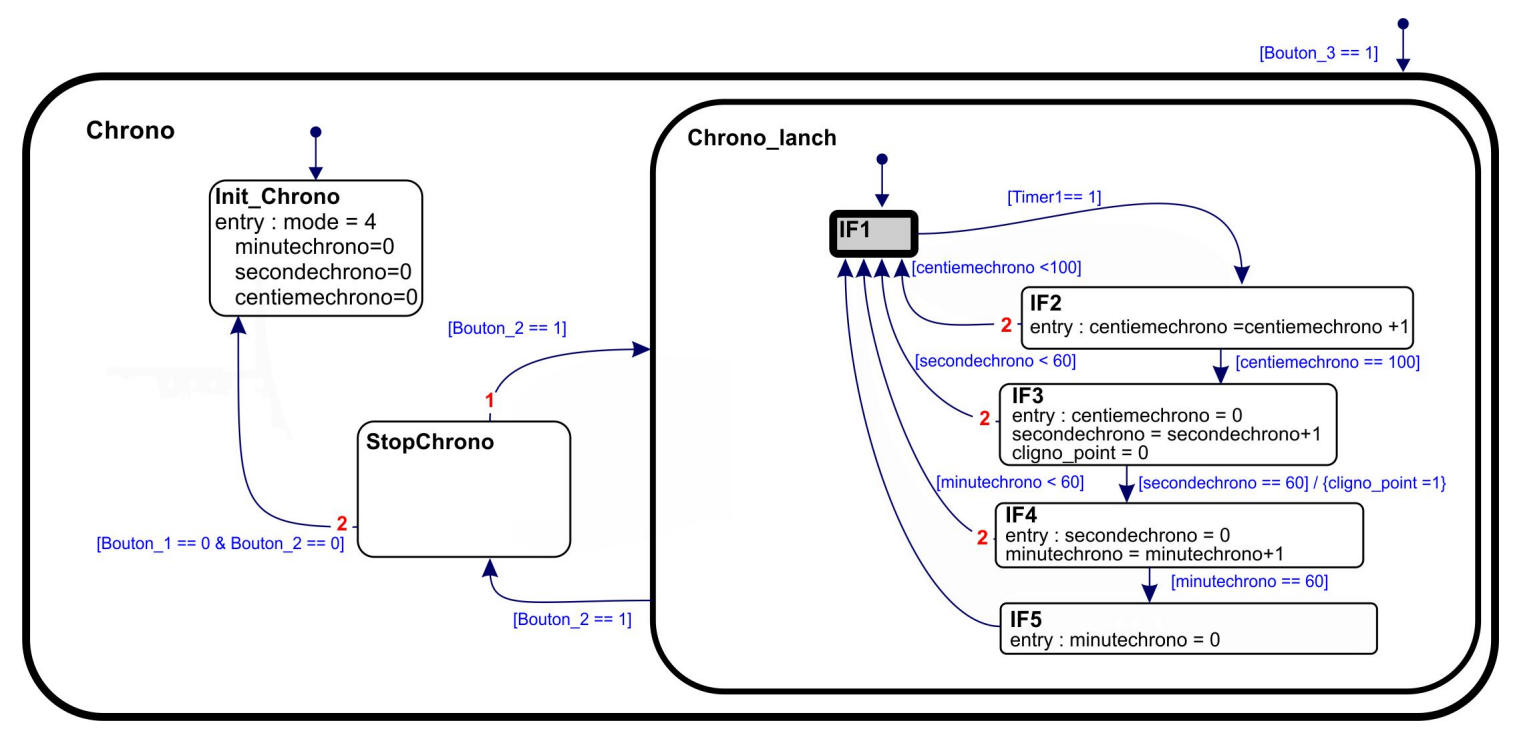

FiguRE 6 - Statechart de la fonction chronomètre

\subsection{Retour d'expérience}

Cette manipulation est proposée dans deux de nos cycles de formation depuis le premier semestre 2009. Cela signifie qu'environ 350 étudiants ont $\mathrm{pu}$ se former aux Statecharts et à la technique de mise en oeuvre que nous leur proposons. Le retour d'expérience commence donc à être suffisamment important pour devenir significatif. Il nous a permis d'apporter diverses améliorations notamment, dans la formulation du travail demandé.

Signalons en premier lieu que l'outil Moodle ([MoODLE, $]$ ) est utilisé pour présenter la maquette et les différentes manipulations à faire. Moodle est l'environnement d'apprentissage libre, maintenant largement répandu dans le monde universitaire et qui est déployé sur l'INSA de Toulouse depuis 2007. Cet outil est notamment intéressant pour diffuser aux étudiants toutes sortes de supports de cours, de TD ou de TP, mais également pour effectuer des évaluations.

Concernant cette manipulation l'emploi de cet outil a un double impact pour les étudiants : la préparation et l'évaluation. Ces deux points sont présentés ci-dessous, en rapportant également les éléments importants retournés par les étudiants lors du sondage anonyme qui leur a été proposé pour évaluer cet enseignement.

Grâce à la plate-forme Moodle, il nous est possible de mettre à la disposition des étudiants plusieurs fichiers de conception initiale (fichier Matalb/Simulink). Accompagnés des explications nécessaires, cela nous permet de leur demander un travail de préparation efficace. Ils doivent en effet s'inspirer des blocs fonction déjà existants pour développer leurs propres blocs. Cette préparation, qui reste relativement simple dans la demande, leur permet de réaliser, lorsqu'ils arrivent en salle de travaux pratiques, la première étape de la manipulation (test de certaines fonctionnalités de la montre déjà modélisées) très rapidement. Le retour des étudiants concernant la prise en main de la manipulation est particulièrement positif. En effet comme les étudiants sont dans la possibilité de voir très rapidement la montre fonctionner, leur niveau de satisfaction est élevé et la motivation pour ajouter de nouvelles fonctionnalités (et donc d'utiliser l'outil Statecharts) est avérée. Le rajout de ces différentes fonctionnalités est proposé de manière incrémentale, 
puisqu'ils ont pu tester leurs blocs aisément et les groupes les plus avancés ont pu développer des fonctionnalités plus poussées, comme par exemple une fonction de chronomètre avec temps intermédiaire. Suite aux demandes des étudiants, nous envisageons de mettre au point un étape de simulation (accessible depuis moodle) de manière à ce que les étudiants puissent tester leur code avant la mise en œuvre réelle et ainsi appréhender toutes les étapes du développement d'un produit.

L'évaluation : l'ensemble des TP est évalué par un $Q C M$ à la fin de la dernière séance. Cette évaluation individuelle permet de déterminer l'implication de chaque étudiant, à l'inverse de compterendus de séance communs. Ce $Q C M$ (sous Moodle) est basé sur un tirage aléatoire de questions. La banque de questions initialement créée par l'équipe enseignante, est étendue au fil des années. Comme le tirage est aléatoire et que notre banque de questions devient conséquente, les étudiants ne sont jamais en face du même questionnaire. Une des difficultés rencontrée par cette méthode d'évaluation, et qui a fait l'objet d'un certain nombre de retours, est l'attention qu'il faut porter à mettre en place des questions de difficulté équivalente. Suite à ce constant nos questions ont été classées en 3 catégories (facile - normale - avancée) et le tirage aléatoire est maintenant programmé pour qu'un nombre équivalent de questions issues de chaque catégorie soit proposé à l'examiné. Nous espérons, avec cette modification, répondre aux attentes des étudiants qui estimaient que le facteur chance avait une influence trop importante sur la note obtenue.

\section{Conclusion Evolutions}

Au travers de cet article, nous avons souhaité présenter un support applicatif simple, attractif et relativement facile à mettre en place permettant d'illustrer d'une part un enseignement de base sur les Statecharts et d'autre part des concepts fondamentaux de commande. Ce projet Montre Digitale est bien entendu perfectible néanmoins il offre d'ores et déjà de nombreuses possibilités pédagogiques. La prochaine évolution envisagée pour ce projet est de voir les possibilités offertes par l'outil Stateflow coder dans l'optique de mettre en place une commande par $\mu$ contrôleur STM32. Ceci permettrait d'établir le lien avec les enseignements de contrôle de périphériques dans lesquels ce $\mu$ contrôleur vient d'être introduit.

\section{Remerciements}

Les auteurs souhaiteraient remercier les étudiants de 4ème année Automatique et Electronique promotion 2009 impliqués dans le projet tutoré "Montre".

\section{Références}

[HAREL, 1988] HAREL, D. (1988). On visual formalisms. Communications of the Association Computing Machinery, 31(5) :514-530.

[Matlab And Simulink, ] Matlab And SimuLINK. Stateflow and stateflow coder 7 user's guide. http ://www.mathworks.com.

[MoOdLE, ] MoOdLE. https ://moodle.insatoulouse.fr/. 